\title{
Clinical course and outcome of SARS-CoV-2 infection in multiple sclerosis patients treated with disease-modifying therapies - the Polish experience
}

Agata Czarnowska ${ }^{1}$, Waldemar Brola ${ }^{2}$, Olga Zajkowska ${ }^{3}$, Stanisław Rusek ${ }^{4}$, Monika Adamczyk-Sowa ${ }^{5}$, Katarzyna Kubicka-Bączyk ${ }^{5}$, Alicja Kalinowska-Łyszczarz ${ }^{6}$, Karolina Kania ${ }^{7}$, Agnieszka Słowik $^{8}$, Marcin Wnuk $^{8}$, Monika Marona ${ }^{8}$, Aleksandra Podlecka-Piętowska ${ }^{9}$, Monika Nojszewska ${ }^{9}$, Beata Zakrzewska-Pniewska ${ }^{9}$, Elżbieta Jasińska ${ }^{2,10}$, Katarzyna Gołuch ${ }^{10}$, Beata Lech ${ }^{11}$, Magdalena Noga ${ }^{11}$, Adam Perenc ${ }^{11}$, Małgorzata Popiel ${ }^{11}$, Anetta Lasek-Bal ${ }^{12}$, Przemysław Puz ${ }^{12}$, Katarzyna Maciejowska ${ }^{12}$, Marta Kucharska-Lipowska ${ }^{13}$, Michał Lipowski ${ }^{14}$, Katarzyna Kapica-Topczewska ${ }^{1}$, Monika Chorąży ${ }^{1}$, Joanna Tarasiuk ${ }^{1}$, Jan Kochanowicz ${ }^{1}$, Joanna Kulikowska ${ }^{1}$, Sławomir Wawrzyniak ${ }^{15}$, Anna Niezgodzińska-Maciejek ${ }^{15}$, Anna Pokryszko-Dragan ${ }^{16}$, Ewa Gruszka ${ }^{16}$, Sławomir Budrewicz ${ }^{16}$, Marta Białek ${ }^{17}$, Iwona Kurkowska-Jastrzębska ${ }^{18}$, Katarzyna Kurowska ${ }^{18}$, Adam Stępieñ ${ }^{19}$, Agata Włodek ${ }^{20}$,

Violetta Ptasznik ${ }^{21}$, Małgorzata Pawełczyk ${ }^{22}$, Piotr Sobolewski ${ }^{23}$, Henryka Lejmel ${ }^{24}$, Katarzyna Strzalińska ${ }^{25}$, Maciej Maciejowski ${ }^{26}$, Andrzej Tutaj ${ }^{27}$, Jacek Zwiernik ${ }^{27,28}$, Anna Litwin ${ }^{27}$, Bożena Lewańczyk ${ }^{29}$, Izabela Paprocka ${ }^{29}$, Beata Zwiernik ${ }^{28}$, Aleksandra Pawlos ${ }^{30}$, Andrzej Borysowicz ${ }^{31}$, Anna Narożnik ${ }^{31}$, Anna Michałowska ${ }^{27},{\text { Krzysztof } \text { Nosek }^{30} \text {, Małgorzata Fudala }}^{32}$, Marta Milewska-Jędrzejczak ${ }^{23}$, Alina Kułakowska ${ }^{1 \star}$, Halina Bartosik-Psujek ${ }^{33 *}$

*Senior authors

${ }^{1}$ Department of Neurology, Medical University of Bialystok, Poland

${ }^{2}$ Collegium Medicum, Jan Kochanowski University, Kielce, Poland

${ }^{3}$ Faculty of Economic Sciences, University of Warsaw, Poland

${ }^{4}$ Department of Neurology, Specialist Hospital Ludwika Rydygiera in Krakow, Poland

${ }^{5}$ Department of Neurology, Faculty of Medical Sciences in Zabrze, Medical University of Silesia in Katowice, Poland

${ }^{6}$ Department of Neurology, Division of Neurochemistry and Neuropathology, Poznan University of Medical Sciences, Poland

${ }^{7}$ Department of Neurology, Poznan University of Medical Sciences, Poland

${ }^{8}$ Department of Neurology, Jagiellonian University Medical College, Krakow, Poland

${ }^{9}$ Department of Neurology, Medical University of Warsaw, Poland

${ }^{10}$ Clinical Centre, Resmedica, Kielce, Poland

\footnotetext{
${ }^{11}$ Neurology Clinic with Brain Stroke Sub-Unit, Clinical Hospital No. 2 in Rzeszow, Poland

${ }^{12}$ Department of Neurology, School of Health Sciences, Medical University of Silesia in Katowice, Poland

${ }^{13}$ Department of Neurology, Specialist Hospital in Konskie, Poland

${ }^{14}$ Department of Urology, Specialist Hospital in Konskie, Poland
}

${ }^{15}$ Department of Neurology, 10th Military Research Hospital and Polyclinic, Independent Public Healthcare Centre, Bydgoszcz, Poland

${ }^{16}$ Department of Neurology, Wroclaw Medical University, Wroclaw, Poland

${ }^{17}$ Department of Neurology, Regional Specialised Hospital No. 4 in Bytom, Poland

${ }^{18}$ 2nd Department of Neurology, Institute of Psychiatry and Neurology, Warsaw, Poland

${ }^{19}$ Department of Neurology, Military Institute of Medicine, Warsaw, Poland

${ }^{20}$ Department of Neurology, Masovian Voivodeship Hospital in Siedlce, Poland 


\author{
${ }^{21}$ Department of Neurology, Specialist Hospital in Pila, Poland \\ ${ }^{22}$ Department of Neurology and Stroke, Medical University of Lodz, Poland \\ ${ }^{23}$ Department of Neurology and Stroke Unit in Sandomierz, Collegium Medicum, Jan Kochanowski University in Kielce, Poland \\ ${ }^{24}$ Department of Neurology, The Regional Hospital in Suwalki, Poland \\ ${ }^{25}$ Department of Neurology, The Regional Hospital in Lomza, Poland \\ ${ }^{26}$ KMK Clinical, MS Centre, Katowice, Poland \\ ${ }^{27}$ Neurology Ward, Provincial Specialist Hospital, Olsztyn, Poland \\ ${ }^{28}$ Department of Neurology, University of Warmia and Mazury, Olsztyn, Poland \\ ${ }^{29}$ Neurology Ward, Provincial Integrated Hospital, Elblag, Poland \\ ${ }^{30}$ Department of Pharmacology and Toxicology, Faculty of Medicine, University of Warmia and Mazury, Olsztyn, Poland \\ ${ }^{31}$ Department of Neurology, Specialist Hospital Dr Tytus Chałubinski, Radom, Poland \\ ${ }^{32}$ Department of Neurology, Regional Hospital in Skarzysko-Kamienna, Poland \\ ${ }^{33}$ Department of Neurology, Institute of Medical Sciences, Medical College of Rzeszow University, Poland
}

\title{
ABSTRACT
}

Background. The aim of this study was to report the course and outcome of SARS-CoV-2 infection in multiple sclerosis (MS) patients treated with disease-modifying therapies (DMTs) in Poland.

A major concern for neurologists worldwide is the course and outcome of SARS-CoV-2 infection in patients with MS treated with different DMTs. Although initial studies do not suggest an unfavourable course of infection in this group of patients, the data is limited.

Materials and methods. This study included 396 MS patients treated with DMTs and confirmed SARS-CoV-2 infection from 28 Polish MS centres. Information concerning patient demographics, comorbidities, clinical course of MS, current DMT use, as well as symptoms of SARS-CoV-2 infection, need for pharmacotherapy, oxygen therapy, and/or hospitalisation, and short-term outcomes was collected up to 30 January 2021. Additional data about COVID-19 cases in the general population in Poland was obtained from official reports of the Polish Ministry of Health.

Results. There were 114 males (28.8\%) and 282 females (71.2\%). The median age was 39 years (IQR 13). The great majority of patients with MS exhibited relapsing-remitting course (372 patients; 93.9\%). The median EDSS was 2 (SD 1.38), and the mean disease duration was 8.95 (IQR 8) years. Most of the MS patients were treated with dimethyl fumarate $(164 ; 41.41 \%)$. Other DMTs were less frequently used: interferon beta $(82 ; 20.70 \%)$, glatiramer acetate $(42 ; 10.60 \%)$, natalizumab $(35 ; 8.84 \%)$, teriflunomide $(25 ; 6.31 \%)$, ocrelizumab $(20 ; 5.05 \%)$, fingolimod $(16 ; 4.04)$, cladribine $(5 ; 1.26 \%)$, mitoxantrone $(3 ; 0.76 \%)$, ozanimod $(3 ; 0.76 \%)$, and alemtuzumab $(1 ; 0.25 \%)$. The overall hospitalisation rate due to COVID-19 in the cohort was $6.81 \%$ (27 patients). Only one patient $(0.3 \%)$ died due to SARS-CoV-2 infection, and three $(0.76 \%)$ patients were treated with mechanical ventilation; 106 (26.8\%) patients had at least one comorbid condition. There were no significant differences in the severity of SARS-CoV-2 infection regarding patient age, duration of the disease, degree of disability (EDSS), lymphocyte count, or type of DMT used.

Conclusions and clinical implications. Most MS patients included in this study had a favourable course of SARS-CoV-2 infection. The hospitalisation rate and the mortality rate were not higher in the MS cohort compared to the general Polish population. Continued multicentre data collection is needed to increase the understanding of SARS-CoV-2 infection impact on the course of MS in patients treated with DMTs.

Key words: multiple sclerosis, COVID-19, SARS-CoV-2, disease-modifying therapies

(Neurol Neurochir Pol 2021; 55 (2): 212-222)

\section{Introduction}

Severe acute respiratory syndrome coronavirus-2 (SARS- $\mathrm{CoV}-2$ ) is an enveloped, single-stranded, RNA virus, and a member of the family Coronaviridae. It was isolated for the first time on 7 January 2020 in China. Since the first unexplained pneumonia cases reported from Wuhan, China in early December 2019, the global spread of the novel coronavirus had resulted in over 100,000,000 confirmed cases and over $2,000,000$ deaths by the end of January $2021[1,2]$. The World 


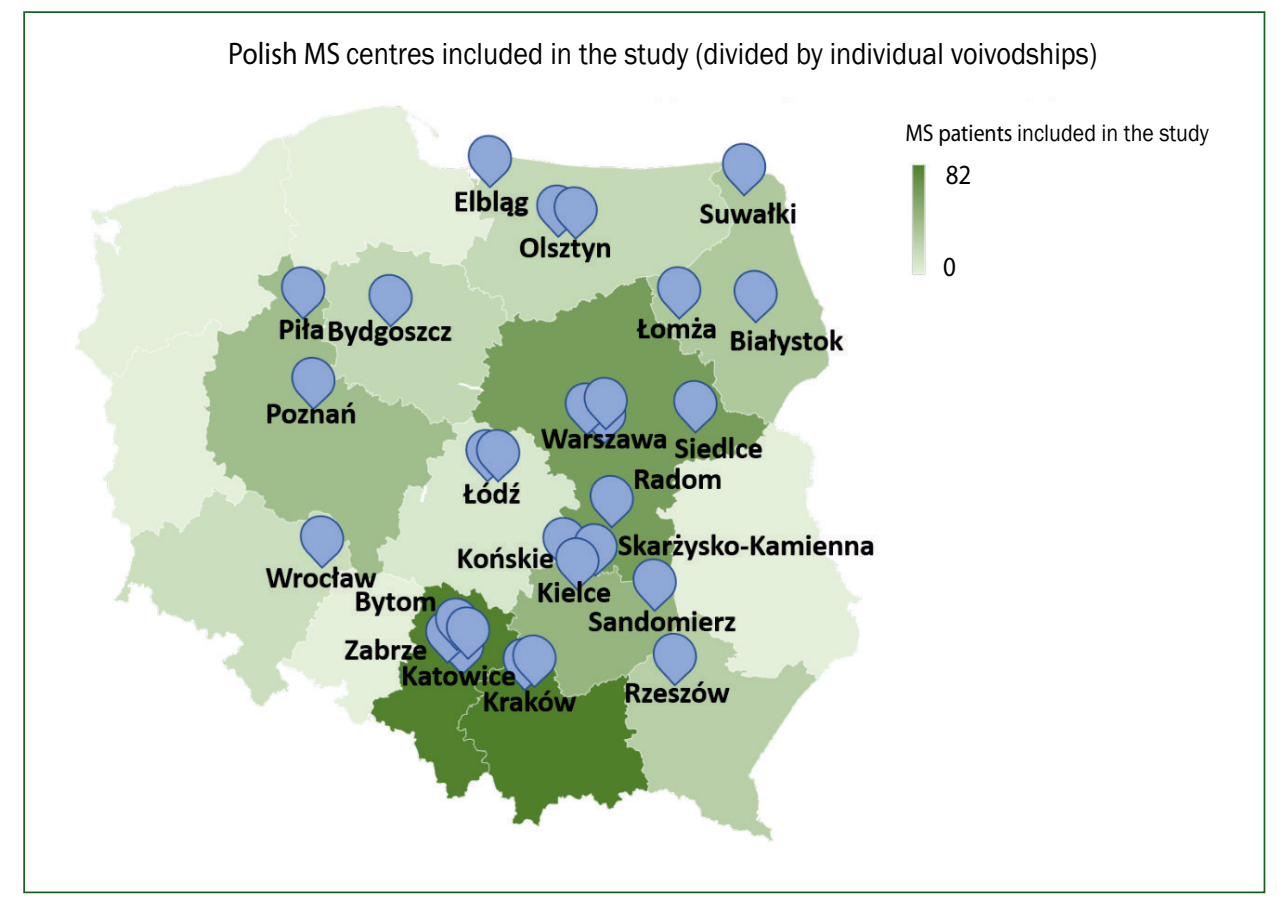

Figure 1. Locations of MS centres participating in study

Health Organisation (WHO) declared a pandemic in March 2020. The current coronavirus outbreak led to a much larger global threat compared to previous outbreaks caused by severe acute respiratory syndrome (SARS-CoV) in late 2002 or the Middle East respiratory syndrome (MERS-CoV) in 2012 [3].

The majority of SARS-CoV-2 infections are asymptomatic. The most common clinical presentation in symptomatic individuals is pneumonia, now termed Coronavirus Disease 2019 (COVID-19). However, the clinical spectrum of SARS- $\mathrm{CoV}-2$ infection is broad, and is a particular threat to those over 60 years of age and those with comorbidities [4]. The virus primarily targets the respiratory tract; however, there is clinical evidence of neuro-invasive properties. According to scientific reports published so far, $18-80 \%$ of patients can develop neurological symptoms such as headache, olfactory and gustatory dysfunctions, encephalopathy, encephalitis and cerebrovascular pathologies, acute myelitis, and Guillain-Barré syndrome $[5,6]$. The exact mechanism by which SARS-CoV-2 infects the central nervous system (CNS) is still under investigation. The entrance points to the CNS for SARS-CoV-2 include retrograde transfer from the olfactory nerve, increased permeability of the blood-brain barrier during the viremia phase, and a synapse-connected route from the peripheral nerve terminal [7].

Neurologists are interested in understanding whether patients with multiple sclerosis (MS) undergoing disease-modifying therapies (DMTs) are more susceptible to developing COVID-19 or worse clinical outcomes. The initial studies do not suggest an unfavourable course of the infection due to the use of most DMTs, although the data is limited [8-10]. Some immunosuppressive therapies may even play a protective role because overactive immune response can lead to clinical deterioration during the course of COVID-19 [11].

To better understand the impact of SARS-CoV-2 infection on the MS population, we collected data from 28 Polish MS centres. The aim of our study was to report symptoms, the course and short-term outcomes of SARS-CoV-2 infection in MS patients treated with different DMTs.

\section{Materials and methods}

Participants were recruited from $28 \mathrm{MS}$ centres from across the country (Fig. 1). The Multiple Sclerosis and Neuroimmunology Section of the Polish Neurological Society published an announcement about the study at www.ptneuro.pl and every MS centre in Poland was invited to participate. The data was obtained by neurologists using a pre-prepared questionnaire (the same for all MS centres). We included patients with a confirmed diagnosis of MS according to the 2010 and 2017 McDonald criteria who were being treated with DMTs. $[12,13]$. Only patients with confirmed SARS-CoV-2 infection were included in the study. The infection was detected by positive polymerase chain reaction (Seegene Allplex $2019 \mathrm{nCoV}$ assay, PCR, SARS-CoV-2 Real Time PCR LAB-KIT"w by BIOMAXIMA S.A., GeneProof SARS-CoV-2 PCR Kit), positive antigen test against SARS-CoV-2 (Panbio ${ }^{\text {mix }}$ COVID-19 Ag Rapid Test, Abbott), or the presence of antibodies against SARS-CoV-2 (EUROIMMUN Anty-SARS-CoV-2 ELISA IgA, IgG). Disability was assessed by the Expanded Disability Status Scale (EDSS) [14]. We collected patient demographics, 


\begin{tabular}{|c|c|c|c|c|c|c|c|}
\hline Demographics & No. & (\%) & Range & Mean & MEDIAN & IQR & SD \\
\hline Number of patients & 396 & 100 & & & & & \\
\hline Age & & & $18-68$ & 40.05 & 39 & 13 & 9.97 \\
\hline Male & 114 & 28.8 & & & & & \\
\hline Female & 282 & 71.2 & & & & & \\
\hline Smokers & 46 & 11.6 & & & & & \\
\hline Clinical characteristics due to MS & No. & (\%) & Range & Mean & MEDIAN & IQR & SD \\
\hline \multicolumn{8}{|l|}{ Disease course } \\
\hline RRMS & 372 & 93.9 & & & & & \\
\hline PPMS & 22 & 5.6 & & & & & \\
\hline SPMS & 2 & 0.5 & & & & & \\
\hline EDSS & & & $0-6.5$ & 2.37 & 2 & 2.5 & 1.38 \\
\hline Disease duration (years) & & & $0-33$ & 8.95 & 8 & 8 & 5.91 \\
\hline Duration of DMTs use (years) & & & 0-19 & 5.79 & 5 & 2 & 4.07 \\
\hline \multicolumn{8}{|l|}{ DMTs: } \\
\hline Interferon beta & 82 & 20.70 & & & & & \\
\hline Glatiramer acetate & 42 & 10.60 & & & & & \\
\hline Dimethyl fumarate & 164 & 41.41 & & & & & \\
\hline Teriflunomide & 25 & 6.31 & & & & & \\
\hline Fingolimod & 16 & 4.04 & & & & & \\
\hline Natalizumab & 35 & 8.84 & & & & & \\
\hline Ocrelizumab & 20 & 5.05 & & & & & \\
\hline Cladribine & 5 & 1.26 & & & & & \\
\hline Alemtuzumab & 1 & 0.25 & & & & & \\
\hline Mitoxantrone & 3 & 0.76 & & & & & \\
\hline Ozanimod & 3 & 0.76 & & & & & \\
\hline
\end{tabular}

MS - multiple sclerosis; SD - standard deviation; RRMS - relapsing remitting multiple sclerosis; PPMS - primary progressive multiple sclerosis; SPMS - secondary progressive multiple sclerosis; EDSS Expanded Disability Status Scale; DMTs — disease-modifying therapies

current DMT use, and information about the course of SARS-CoV-2 infection (symptoms, need for hospitalisation, oxygen therapy, pharmacotherapy and short-term outcome). Data was collected up to 30 January 2021. Additional data about COVID-19 cases in the general Polish population was obtained from official reports of the Polish Ministry of Health [15].

Demographics, MS duration, level of disability, DMT use, method of SARS-CoV-2 infection confirmation, symptoms, number of hospitalisations, treatment during SARS-CoV-2 infection, and key comorbidities were reported with descriptive statistics, using range, mean and standard deviation or median and interquartile range depending on the data type. For between-group comparisons, a parametric two-sided t-test, Fisher's exact p-test, and a nonparametric Mann-Whitney test were used. We compared age, duration of the disease, EDSS, DMT use, and the presence of comorbid diseases between patients who required oxygen therapy/hospitalisation and the rest of the cohort. Multivariate analysis and univariate analysis were used to evaluate the odds ratio for hospitalisation according to treatment with different DMTs. All calculations were performed with STATA 15 software (StataCorp 2017) [16].

The study was approved (approval No. 6/2021) by the Bioethics Committee at Collegium Medicum, Jan Kochanowski University in Kielce, Poland.

\section{Results}

The study finally included information about 396 MS patients with confirmed SARS-CoV-2 infection and treated with different DMTs. Demographics and clinical characteristics of the study group are set out in Table 1 .

There were 106 (26.8\%) patients with at least one comorbid condition: 10 had two, and two subjects had three comorbid diseases. The most common comorbidities were: hypertension (44 patients; $11.1 \%$ of the cohort), diabetes in 12 (3\%) patients, coronary artery disease in six (1.5\%), asthma in nine (2.3\%), and chronic liver disease in four (1\%); 55 (13.9\%) patients had one other comorbidity (chronic obstructive pulmonary 
Table 2. Number of SARS-CoV-2 infection diagnoses confirmed by different diagnostic tests

$\begin{array}{lcc} & \text { No. of patients } & \text { Percentage of cohort } \\ \text { Positive PCR } & 333 & 23 \\ \text { Positive antigen test confirmed by PCR } & 23 & 5.8 \\ \text { Positive PCR/antigen test and presence of antibodies against SARS-CoV-2 } & 30 \\ \text { Positive antigen test against SARS-CoV-2 } & 39 \\ \text { Presence of antibodies against SARS-CoV-2 } & 36 & 9.9\end{array}$

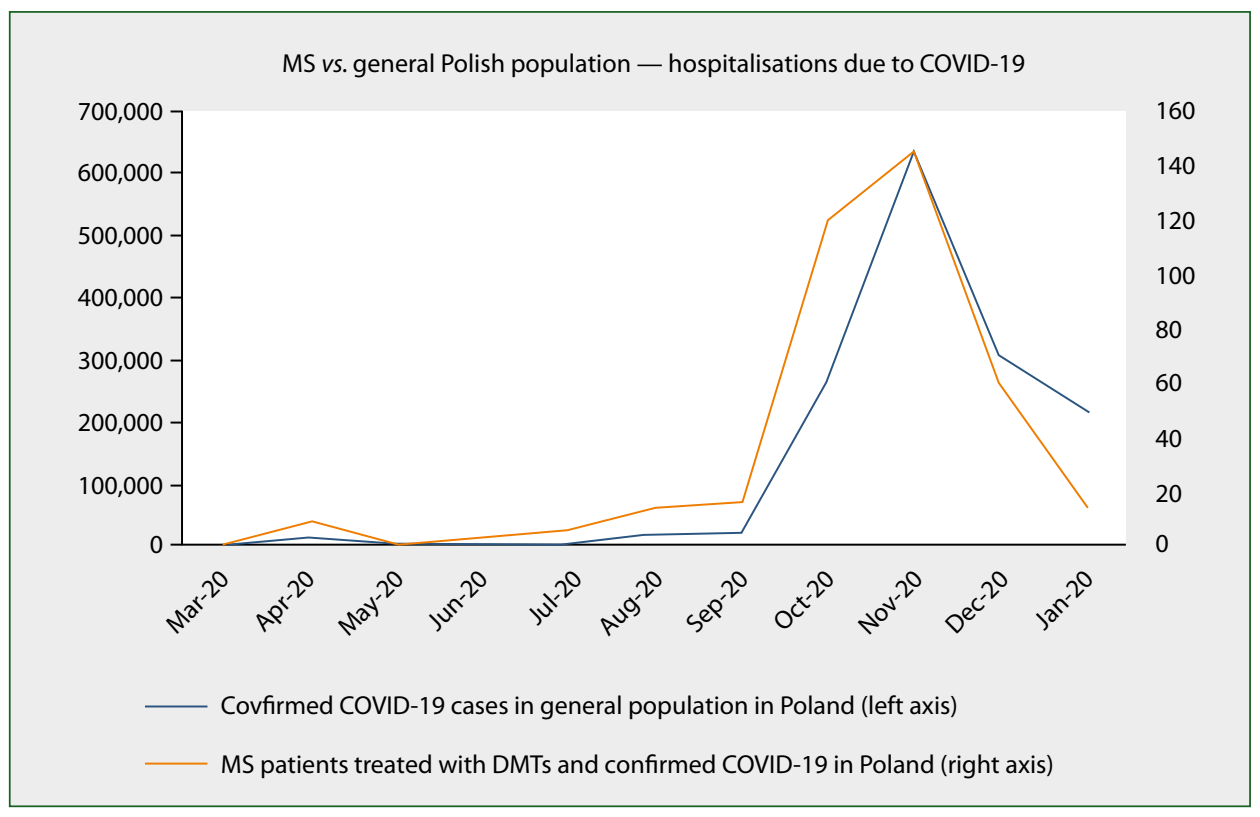

Figure 2. Confirmed cases of COVID-19: MS vs. general Polish population

disease, neoplastic disease, depression, autoimmune diseases of the thyroid gland, etc.). There was no statistically significant difference in the percentage of hospitalised patients between groups with and without comorbidities (Fisher's exact $=0.555)$.

In 332 cases ( $84 \%)$, the infection was confirmed by PCR tested on a nasopharyngeal swab, in 39 (9.9\%) cases the antigen test was positive, and $36(9.1 \%)$ patients had antibodies against SARS-CoV-2. A combination of different tests was used in some subjects (Tab. 2).

The vast majority of the MS cohort was diagnosed with SARS-CoV-2 infection between October and December 2020. The peak incidence of COVID-19 in the MS cohort generally overlapped with a trend in the general population in Poland (Fig. 2). A similar trend was observed with regard to hospitalisation (Fig. 3). We found that 264 (66.7\%) patients reported contact with someone infected with SARS-CoV-2, 82 (20.7\%) patients denied contact with an infected person, and $50(12.6 \%)$ cases had no information about any possible contact.
The overall hospitalisation rate due to COVID-19 in the cohort was $6.81 \%$ (27 patients). Only one patient $(0.3 \%)$ died due to COVID-19 infection, and three patients were treated with mechanical ventilation. The hospitalisation rate due to COVID-19 in the general Polish population was $7.98 \%$, and the mortality rate was $2.46 \%$ [15]. The characteristics of hospitalised individuals in the MS cohort are set out in Table 3. The distribution of DMTs in the group of hospitalised patients and those who were not hospitalised is set out in Figure 4 and Table 4.

In our observation, patients treated with ocrelizumab were the most likely to be hospitalised while being infected with SARS-CoV-2 ( $\mathrm{p}=0.004)$. We did not consider interferon beta, glatiramer acetate, or dimethyl fumarate as increasing hospitalisation probability because univariate analysis did not confirm the significance of the result; 48 (12.1\%) patients interrupted DMTs therapy during SARS-CoV-2 infection, and of these nine were hospitalised. A Mann-Whitney test showed no significant difference in disability (EDSS) between hospitalised and non-hospitalised patients in the cohort $(\mathrm{p}=0.08211)($ Fig. 5) . 


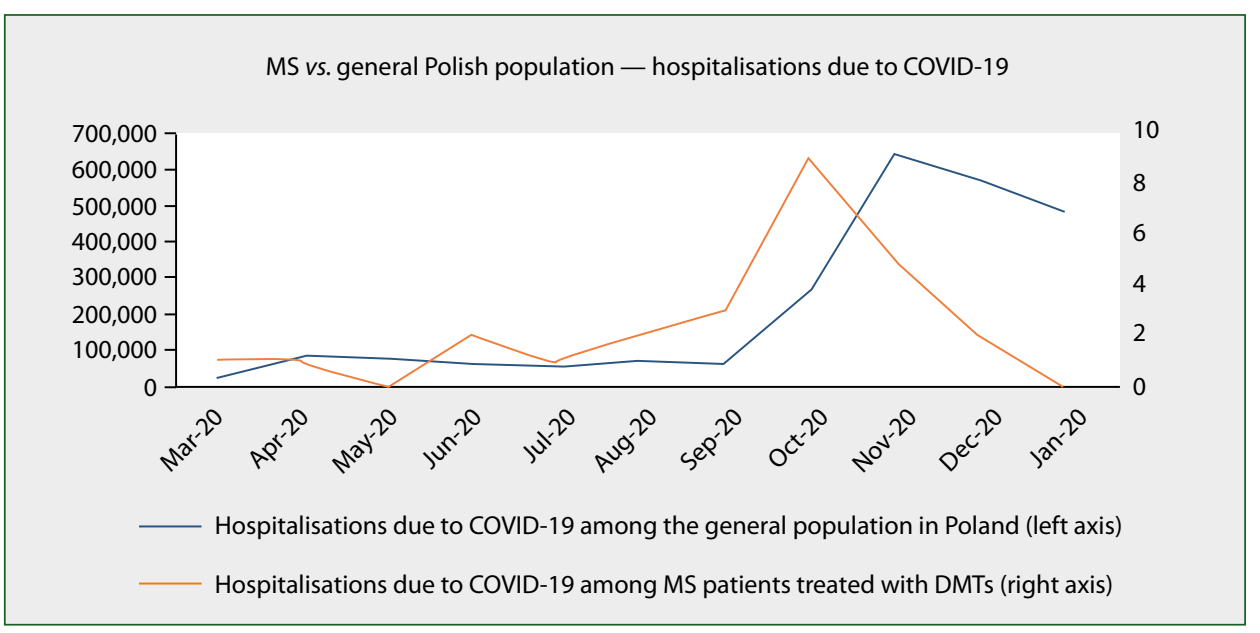

Figure 3. Hospitalisations due to COVID-19: MS vs. general Polish population

Table 3. Characteristics of hospitalised MS patients

$\begin{array}{lc}\text { Characteristics of hospitalised patients } & \text { No. (\%) } \\ \text { Number } & 27(100) \\ \text { Hospitalisation }>10 \text { days } & 12(44.4) \\ \text { Hospitalisation < } 10 \text { days } & 15(55.5) \\ \text { Passive oxygen therapy } & 12(44.4) \\ \text { Mechanical ventilation } & 3(11.1) \\ \text { Deaths } & 1(3.7) \\ \text { Treatment } & \\ \text { Remdesivir } & 3(11.1) \\ \text { Glucocorticosteroids } & 6(22.2) \\ \text { Convalescent plasma therapy } & 8(29.6) \\ \text { Antibiotics } & 23(85.2)\end{array}$

The mean age of hospitalised patients was 42.42 years, and the mean age of non-hospitalised patients was 39.88 . A two-sided t-test suggested that this difference was not statistically significant $(\mathrm{p}=0.209)$ (Fig. 6). The patients who were in need of oxygen therapy during COVID-19 were on average 6.17 years older than those who did not require oxygen support $(\mathrm{p}=0.0227)$.

Laboratory findings in the MS cohort are set out in Table 5. There was no significant difference in the rate of hospitalisation between patients with and without lymphopenia (Fisher's exact $=0.400$ ). Patients with lymphopenia also did not require oxygen therapy (passive and active oxygen therapy) more often than did patients without lymphopenia (Fisher's exact $=1.000$ ). Symptoms presented during COVID-19 infection are set out in Table 6.

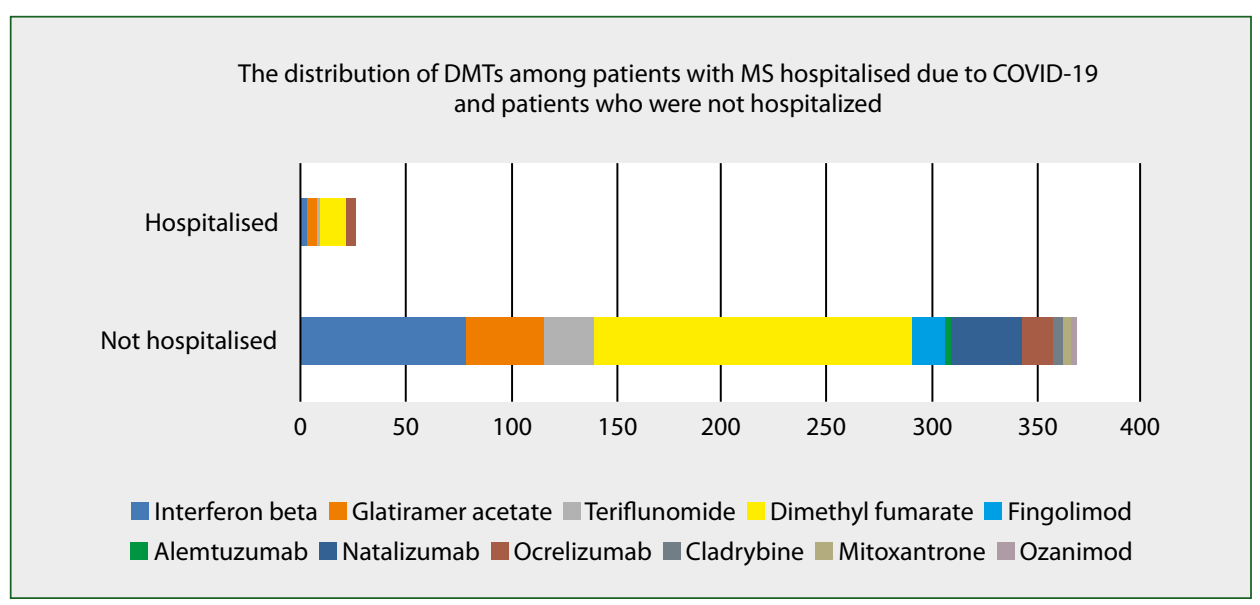

Figure 4. Distribution of DMTs among hospitalised and non-hospitalised MS patients 
Table 4. Distribution of DMTs among hospitalised and non-hospitalised MS patients

$\begin{array}{lccc}\text { DMT } & \begin{array}{c}\text { Non-hospitalised } \\ \text { patients no. }\end{array} & \begin{array}{c}\text { Hospitalised } \\ \text { patients no. }\end{array} & \begin{array}{c}\text { Percentage of patients hospitalised on particular DMT } \\ \text { in relation to all patients treated with particular DMT }\end{array} \\ \begin{array}{l}\text { Interferon beta } \\ \text { Glatiramer acetate }\end{array} & 37 & 5 & 3.66(3 \text { out of } 82) \\ \text { Dimethyl fumarate } & 151 & 13 & 7.90(5 \text { out of } 42) \\ \text { Teriflunomide } & 24 & 1 & 0.04(1 \text { out of 25) } \\ \text { Fingolimod } & 16 & 0 & 0 \\ \text { Natalizumab } & 35 & 0 & 0 \\ \text { Ocrelizumab } & 15 & 5 & 25(5 \text { out of 20) } \\ \text { Cladribine } & 5 & 0 & 0 \\ \text { Alemtuzumab } & 1 & 0 & 0 \\ \text { Mitoxantrone } & 3 & 0 & 0\end{array}$

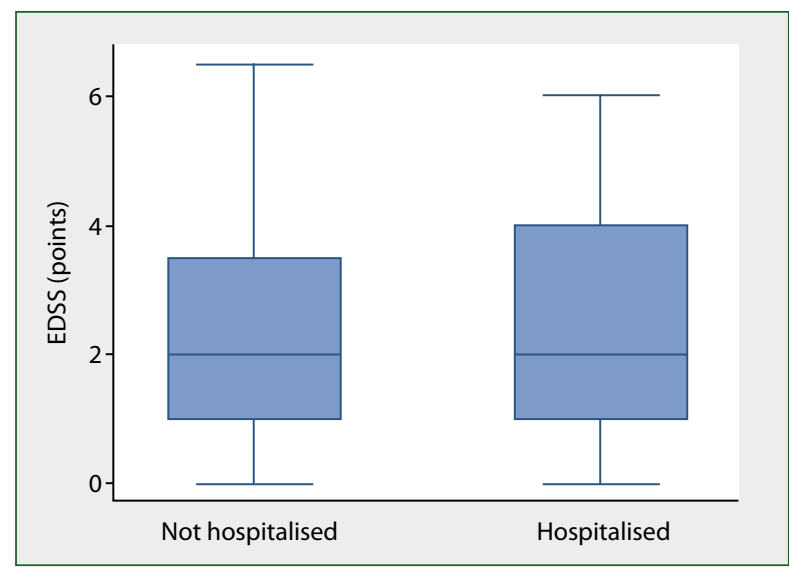

Figure 5. Distribution of EDSS among hospitalised and non-hospitalised patients in MS cohort

Table 5. Laboratory findings among MS cohort during SARS-CoV-2 infection

\begin{tabular}{lll}
$\begin{array}{l}\text { Laboratory findings during } \\
\text { COVID-19 }\end{array}$ & \multicolumn{1}{c}{$\begin{array}{c}\text { No. of } \\
\text { patients }\end{array}$} & $\begin{array}{c}\text { Percentage } \\
\text { of cohort }\end{array}$ \\
\hline $\begin{array}{l}\text { Lymphopenia } \\
\left(<1 \times 10^{3} / \mu \mathrm{L}\right)\end{array}$ & 69 & 17.4 \\
No lymphopenia & 283 & 71.5 \\
Lymphocytes result unknown & 44 & 11.1 \\
Leukopenia $\left(<3 \times 10^{3} / \mu \mathrm{L}\right)$ & 13 & 3.3 \\
No leukopenia & 338 & 85.4 \\
WBC result unknown & 45 & 11.4 \\
\hline
\end{tabular}

COVID-19 - coronavirus disease 2019; WBC - white blood cells

\section{Discussion}

To the best of our knowledge, this is the largest cohort of Polish MS patients with concomitant SARS-Cov-2 infection.

Our study showed that the outcome of COVID-19 in the group of MS patients treated with DMTs compared to the

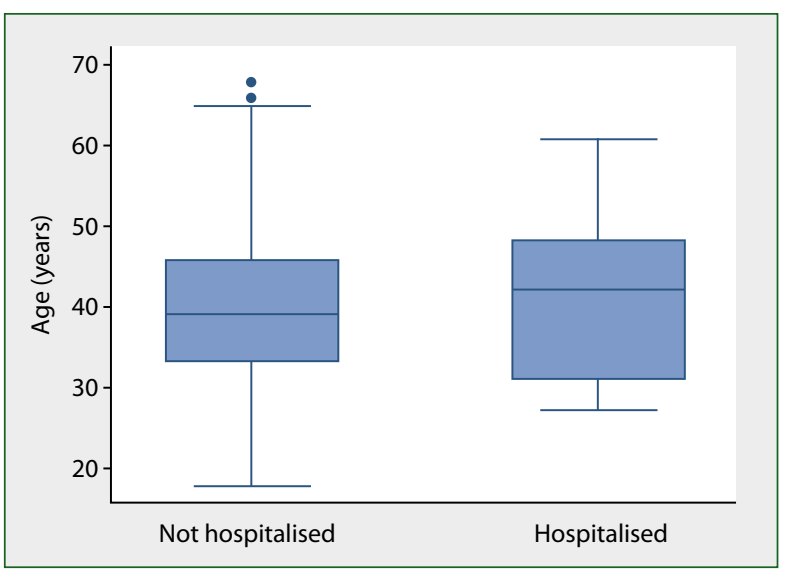

Figure 6. Distribution of age among hospitalised and non-hospitalised patients in MS cohort

general population was favourable. The SARS-CoV-2 infection among MS patients treated with DMTs is a concern for neurologists. The disease itself, and the use of immunomodulatory and immunosuppressive therapies, raise questions regarding the safety of MS patients during the pandemic. The cause and effect relationship between MS or accelerated neurodegeneration and viral infections has been under discussion for a long time $[17,18]$. Recently, a nationwide study from Sweden showed that patients with MS are generally at a greater risk of infections [19]. Safety concerns among Polish MS patients is underlined by the fact that the prevalence of MS in Poland is tending to increase [20,21].

Our findings suggest favourable infection outcomes in multiple sclerosis patients treated with different DMTs. Most patients had mild symptoms and did not require hospitalisation. Only 27 (6.81\%) patients required hospitalisation, and only one patient $(0.3 \%)$ died due to COVID-19 infection; three $(0.76 \%)$ patients were treated with mechanical ventilation.

The second wave of COVID-19 in Poland emerged in early November 2020, and most cases of SARS-CoV-2 infection in 
Table 6. Symptoms presented among patients with MS treated with DMTs during SARS-CoV-2 infection

\begin{tabular}{lcc}
$\begin{array}{l}\text { Symptoms during } \\
\text { SARS-CoV-2 infection }\end{array}$ & $\begin{array}{c}\text { Percentage } \\
\text { of cohort }\end{array}$ & $\begin{array}{c}\text { Risk of } \\
\text { hospitalisation } \\
\text { (odds ratio) }\end{array}$ \\
\hline Fever & 56.2 & 1.921 \\
Fatigue & 40.5 & 1.004 \\
Loss of smell & 36.5 & 0.593 \\
Muscle pain & 35.7 & 1.471 \\
Cough & 34.9 & 1.791 \\
Headache & 28.1 & 0.709 \\
Bone and joint pain & 23.8 & 0.715 \\
Chills & 12.4 & 1.252 \\
Loss of taste & 12.2 & 1.723 \\
Sore throat & 11.6 & 0.923 \\
Swelling of nasal mucosa & 10.6 & 1.000 \\
Shortness of breath & 7.1 & 7.347 \\
Asymptomatic & 5.6 & 0.637 \\
Rash & 1.8 & 2.327 \\
Diarrhoea & 1.5 & 1.000 \\
Pneumonia & 0.3 & 1.000 \\
Vomiting & 0.3 & 1.000 \\
Abdominal pain & 0.3 & 1.000 \\
& & \\
\hline & &
\end{tabular}

the MS cohort occurred during that time. The peak of MS patient hospitalisations was in October 2020, a month earlier than the peak of all hospitalisations due to COVID-19 in Poland (Fig. 3). It seems that at the beginning of the second wave of COVID-19 in Poland, doctors' decisions to hospitalise MS patients with SARS-CoV-2 infection might have been determined largely due to the MS coexistence, rather than by the patient's actual clinical condition. Initial observations on the favourable course of SARS-CoV-2 infection in MS patients meant that patients with MS were hospitalised less frequently than the general population at later periods. Finally, the hospitalisation rate and the mortality rate were slightly lower in our MS cohort compared to the general Polish population $(6.81 \%$ vs. $7.98 \%$ and $0.3 \%$ vs. $2.46 \%$, respectively).

The initial observational studies also suggest that patients with MS treated with DMTs are generally not at greater risk of severe COVID-19 infection [22-25]. However, in an Iranian MS cohort, the incidence of COVID-19 was comparable to the general population, but the hospitalisation rate was significantly higher [26]. On the other hand, preliminary reports on Italian MS patients, as well as Brazilian and Chilean observations, showed that most patients with MS exhibit a mild course of SARS-CoV-2 infection despite the maintenance of DMTs $[9,27,28]$. The vast majority of our patients $(87.9 \%)$ also continued treatment during their SARS-CoV-2 infection. Similar observations indicating no association between immunotherapy and the risk of COVID-19 were found in a group of more than 2,000 patients with NMOSD under appropriate immunotherapy in China. In this group, only two patients developed COVID-19 [29].

The favourable course of SARS-CoV-2 infection in MS patients could be due to the fact that most of them are younger and have fewer comorbidities than do patients from the general population. People aged over 60 and with multiple comorbidities often have severe disease [30,31]. The median age in our cohort was 39 . Age did not significantly differ between hospitalised and non-hospitalised patients with MS. However, the mean age of patients who required oxygen therapy was higher than those who did not require oxygen support. In a French multicentre study of 347 patients with MS who developed COVID-19, age, obesity, and MS-related disability were independent risk factors for more severe COVID-19, but DMT exposure was not associated with COVID-19 severity [22]. In our study, the degree of disability as assessed by EDSS was similar in hospitalised and non-hospitalised patients, but patients included in our study were in the early stage of the disease, with a median EDSS of 2.0.

We found no association between comorbid diseases and the severity of SARS-CoV-2 infection. Most of our MS patients (more than 70\%) had no comorbidities. Only one patient who required hospitalisation and oxygen therapy had more than one comorbid disease: hypertension and chronic obstructive pulmonary disease. Chronic pulmonary diseases are known to be a strong risk factor for serious COVID-19 [32]. Another risk factor for a severe clinical course of SARS-CoV-2 infection is diabetes mellitus [33] Among 12 (3\%) patients with diabetes in our cohort, one required hospitalisation due to low saturation and unilateral pneumonia. Two patients in our cohort had three comorbid diseases although none of them required hospitalisation during SARS-CoV-2 infection. We emphasise that comorbid diseases, known to be a worse prognostic factor for COVID-19, were only present in a minority of our patients, probably due to the young age of our cohort which consisted mainly of RRMS patients treated with DMTs.

In Poland, from the beginning of the pandemic, only symptomatic patients were tested for SARS-CoV-2. Therefore, the MS cohort in our study consisted mostly of those who had symptoms of COVID-19. In a few asymptomatic patients, the infection was detected for professional reasons or before a planned hospitalisation.

The most common symptoms of SARS-CoV-2 infection presented in the Polish MS cohort were fever, fatigue, cough and hyposmia. These symptoms were similar to those reported from multicentre studies of general populations worldwide [35]. The course of the disease did not differ greatly between patients with MS and the general population. About $20 \%$ of our subjects denied contact with an infected individual. This shows that asymptomatic carriers can be a major threat [34].

Among the MS cohort, we registered one death during the course of COVID-19. The patient with a fatal outcome was 
a 51-year-old female non-smoker with comorbid hypertension. She had been diagnosed with relapsing-remitting multiple sclerosis 19 years prior to infection. Her EDSS was 6.0, and she has been treated with ocrelizumab for six years (no other immunomodulatory treatment was used before). The last dose of ocrelizumab was given in February 2020, and she was diagnosed with COVID-19 in August 2020 (at the moment of COVID-19 diagnosis there was no lymphopenia).

In the study group, three patients required mechanical ventilation (one male, two female). Interestingly, they were younger (31, 38, and 39 years) and did not suffer from any comorbid diseases. Maximal EDSS in this group was 4.0, and the longest duration of the disease was eight years. They were treated with glatiramer acetate, interferon beta, and dimethyl fumarate, respectively.

One major concern for neurologists treating MS patients with SARS-CoV-2 infection is the safety of B-cell depleting therapies. B-cells are responsible for generating neutralising antibodies. Therefore, there is uncertainty regarding the clinical course of the infection and the effectiveness of the reaction to future vaccination. Most data does not support higher susceptibility toward more severe SARS-CoV-2 infection among patients treated with B-cell depleting therapies [22, 23, 36, 37]. However, Sormani et al. showed an increased risk of severe COVID-19 disease in patients treated with an anti-CD20 agent [38]. Sharifian-Dorche et al. recently reviewed almost 2,500 MS patients with COVID-19 from different studies. They found that patients treated with anti-CD20 agents (ocrelizumab, rituximab) had the highest mortality rate during the infection [39]. In our cohort, patients treated with ocrelizumab had a higher risk of hospitalization due to SARS-CoV-2 infection. However, three out of five patients hospitalized theated with these therapy suffered form PPMS. Our observation of patients with COVID-19 while being on ocrelizumab treatment is limited to 20 individuals, and thus we are not drawing any definite conclusions. Nonetheless, we draw attention to the need to closely monitor patients treated with anti-CD20 agents during SARS-CoV-2 infection.

Patients with progressive forms of MS are more disabled and tend to have a more severe clinical course of SARS-CoV-2 infection [22, 40]. Here, 24 (6\%) patients were diagnosed with progressive MS: 22 patients with PPMS and two with SPMS. Among patients with progressive MS subtypes, four required hospitalisation and passive oxygen therapy. The statistical difference in the course of SARS-CoV-2 infection between RRMS and progressive phenotypes of the disease was not significant in our study. The numbers of patients with PPMS and SPMS were limited, and final conclusions should only be drawn from a larger observation.

Importantly, MS patients with lymphopenia were not hospitalised more often than patients without lymphopenia. This information is an additional indication for how to consider DMT continuation in real-world practice. Our findings are consistent with the initial Dutch experience, but far more data is needed to be certain that lymphopenia is not associated with a more severe course of the disease [41].

In our MS cohort, any obvious advantage of one drug over another was not found in hospitalised patients or in those who needed oxygen therapy. Some studies have shown a trend for an increased risk of infection with immune-resetting drugs versus injectables, but no final conclusions were drawn because of the small number of observations [42]. In our study, none of the $35 \mathrm{MS}$ patients treated with natalizumab required hospitalisation or oxygen therapy. Only one of the 25 patients treated with teriflunomide and only three of the 82 patients treated with interferon beta were hospitalised.

Some studies have shown a potential protective role of interferons in COVID-19, as these are natural antiviral and anti-inflammatory proteins $[43,44]$. There are also studies pointing to some antiviral activity of teriflunomide [45]. In our MS cohort, the percentage of patients treated with interferons beta $(20.70 \%$ of all patients) in relation to those treated with dimethyl fumarate ( $41.41 \%$ of all patients) is much lower than in the entire Polish MS population. In Poland, dimethyl fumarate and interferons beta are used by similar numbers of MS patients (5,939 and 6,095 patients in 2019 respectively). In our MS cohort, patients were most frequently treated with dimethyl fumarate, and 13 of them (7.93\% of the dimethyl group) needed hospitalisation. However, our cohort is not large enough to draw conclusions about the use of DMT and any protective or non-protective role during SARS-CoV-2 infection.

A potential source of bias in our study is that we included only patients who were treated in specialised MS centres. Also, immobile patients with a high degree of motor and/ or cognitive disability were not included in our study, and in that group the outcomes could potentially be worse. Finally, we could not assess differences between treated versus non-treated MS patients, since our study group only included subjects on DMTs.

\section{Conclusions}

Our study did not provide evidence of a more severe course of COVID-19 in a group of Polish patients treated with several DMTs. Most patients did not require hospitalisation or oxygen therapy. Only one patient died. We did not observe a significant difference in the severity of SARS-CoV-2 infection regarding age, MS duration, the degree of disability as measured by EDSS, presence of comorbidities, lymphocyte count, or the use of different DMTs. There is an urgent need to continue multicentre data collection to increase the understanding of SARS-CoV-2 infection impact on MS course in patients treated with DMTs, especially concerning the long-term complications after SARS-CoV-2 infection.

Funding: This publication was prepared without any external source of funding. 
Conflicts of interest: Alina Kułakowska, Waldemar Brola, Halina Bartosik-Psujek, Anna Pokryszko-Dragan, Monika Adamczyk-Sowa, Iwona Kurkowska-Jastrzębska, Katarzyna Kurowska, Katarzyna Kubicka-Baczyk, Beata Zakrzewska-Pniewska, Monika Nojszewska, Elżbieta Jasińska, Aleksandra Podlecka-Piętowska, Karolina Kania and Monika Marona received compensation for speaking and consulting services from Biogen, Bayer, Novartis, Roche, Merck, Teva, and Sanofi-Genzyme. Agnieszka Stowik and Marcin Wnuk received compensation for speaking and consulting services from Biogen, Bayer, Novartis, Roche, Merck, Teva, and Sanofi-Genzyme. They received also a grant from NCBIR ( $n$ r SZPITALE-JEDNOIMIENNE/18/2020). None of the consulting agreements are relevant to the submitted work. Alicja Kalinowska-Lyszczarz received grant funding from Novartis and received compensation for speaking and consulting services from Biogen, Bayer, Novartis, Roche, Merck, Teva, CSL Behring, Shire, and Sanofi-Genzyme. Piotr Sobolewski has had lectureship fees covered by Boehringer-Ingelheim, Ever Pharma, Allergan and travel expenses to scientific conferences covered by Boehringer-Ingelheim, Ipsen and Ever Pharma and Angels Initiative. None of the agreements are relevant to the submitted work.

\section{References}

1. Mao L, Jin H, Wang M, et al. Neurologic Manifestations of Hospitalized Patients With Coronavirus Disease 2019 in Wuhan, China. JAMA Neurol. 2020; 77(6): 683-690, doi: 10.1001/jamaneurol.2020.1127, indexed in Pubmed: 32275288.

2. Coronavirus disease (COVID-19). https://www.who.int/emergencies/ diseases/novel-coronavirus-2019 (2021 Jan 29).

3. Zheng J. SARS-CoV-2: an Emerging Coronavirus that Causes a Global Threat. Int J Biol Sci. 2020; 16(10): 1678-1685, doi: 10.7150/ ijbs.45053, indexed in Pubmed: 32226285.

4. Onder G, Rezza G, Brusaferro S. Case-Fatality Rate and Characteristics of Patients Dying in Relation to COVID-19 in Italy. JAMA. 2020; 323(18): 17751776, doi: 10.1001/jama.2020.4683, indexed in Pubmed: 32203977.

5. Yachou Y, El Idrissi A, Belapasov V, et al. Neuroinvasion, neurotropic, and neuroinflammatory events of SARS-CoV-2: understanding the neurological manifestations in COVID-19 patients. Neurol Sci. 2020; 41(10): 2657-2669, doi: 10.1007/s10072-020-04575-3, indexed in Pubmed: 32725449.

6. Lechien J, Chiesa-Estomba C, Siati DDe, et al. Olfactory and gustatory dysfunctions as a clinical presentation of mild-to-moderate forms of the coronavirus disease (COVID-19): a multicenter European study. European Archives of Oto-Rhino-Laryngology. 2020; 277(8): 2251-2261, doi: 10.1007/s00405-020-05965-1.

7. Haider A, Siddiqa A, Ali N, et al. COVID-19 and the Brain: Acute Encephalitis as a Clinical Manifestation. Cureus. 2020; 12(10): e10784, doi: 10.7759/cureus.10784, indexed in Pubmed: 33154851.

8. Giovannoni G, Hawkes C, Lechner-Scott J, et al. The COVID-19 pandemic and the use of MS disease-modifying therapies. Mult Scler Relat Disord. 2020; 39: 102073, doi: 10.1016/j.msard.2020.102073, indexed in Pubmed: 32334820.

9. Sormani M. An Italian programme for COVID-19 infection in multiple sclerosis. The Lancet Neurology. 2020; 19(6): 481-482, doi: 10.1016/s1474-4422(20)30147-2.
10. Rostami Mansoor S, Ghasemi-Kasman M. Impact of disease-modifying drugs on the severity of COVID-19 infection in multiple sclerosis patients. J Med Virol. 2021; 93(3): 1314-1319, doi: 10.1002/ jmv.26593, indexed in Pubmed: 33044760.

11. Novi G, Mikulska M, Briano F, et al. COVID-19 in a MS patient treated with ocrelizumab: does immunosuppression have a protective role? Mult Scler Relat Disord. 2020; 42: 102120, doi: 10.1016/j. msard.2020.102120, indexed in Pubmed: 32315980.

12. Polman $\mathrm{CH}$, Reingold SC, Banwell B, et al. Diagnostic criteria for multiple sclerosis: 2010 revisions to the McDonald criteria. Ann Neurol. 2011; 69(2): 292-302, doi: 10.1002/ana.22366, indexed in Pubmed: 21387374.

13. Thompson A, Banwell B, Barkhof $F$, et al. Diagnosis of multiple sclerosis: 2017 revisions of the McDonald criteria. The Lancet Neurology. 2018; 17(2): 162-173, doi: 10.1016/s1474-4422(17)30470-2.

14. Kurtzke JF. Rating neurologic impairment in multiple sclerosis: an expanded disability status scale (EDSS). Neurology. 1983; 33(11): 1444-1452, doi: 10.1212/wnl.33.11.1444, indexed in Pubmed: 6685237.

15. Raport zakażeń koronawirusem (SARS-CoV-2) - Koronawirus: informacje i zalecen. https://www.gov.pl/web/koronawirus/wykaz-zarazen-koronawirusem-sars-cov-2 (2021 Feb 5).

16. StataCorp. 2017. Stata Statistical Software: Release 15. College Station, TX: StataCorp LLC. https://www.stata.com/support/fags/resources/citing-software-documentation-faqs/ (2021 Feb 5).

17. Czarnowska A, Kapica-Topczewska K, Zajkowska O, et al. Herpesviridae Seropositivity in Patients with Multiple Sclerosis: First Polish Study. Eur Neurol. 2018; 80(5-6): 229-235, doi: 10.1159/000496402, indexed in Pubmed: 30661064.

18. Czupryna P, Tarasow E, Moniuszko-Malinowska A, et al. MRI and planimetric CT follow-up study of patients with severe tick-borne encephalitis. Infect Dis (Lond). 2016; 48(1): 74-81, doi: 10.3109/23744235.2015.1083119, indexed in Pubmed: 26414745.

19. Castelo-Branco A, Chiesa F, Conte S, et al. Infections in patients with multiple sclerosis: A national cohort study in Sweden. Mult Scler Relat Disord. 2020; 45: 102420, doi: 10.1016/j.msard.2020.102420, indexed in Pubmed: 32736217.

20. Brola W, Sobolewski P, Flaga S, et al. Increasing prevalence and incidence of multiple sclerosis in Poland. Neurol Neurochir Pol. 2017; 51(1): 82-85, doi: 10.1016/j.pjnns.2016.11.005, indexed in Pubmed: 27889289.

21. Wawrzyniak S, Koziarska D, Kułakowska A, et al. Early predictors of injectable disease modifying drugs suboptimal response based on clinical and radiological data assessment in Polish Multiple Sclerosis patients. Neurol Neurochir Pol. 2019; 53(2): 131-137, doi: 10.5603/ PJNNS.a2019.0010, indexed in Pubmed: 30855704.

22. Louapre C, Collongues N, Stankoff B, et al. Covisep investigators. Clinical Characteristics and Outcomes in Patients With Coronavirus Disease 2019 and Multiple Sclerosis. JAMA Neurol. 2020; 77(9): 1079-1088, doi: 10.1001/jamaneurol.2020.2581, indexed in Pubmed: 32589189.

23. Parrotta E, Kister I, Charvet L, et al. COVID-19 outcomes in MS: Observational study of early experience from NYU Multiple Sclerosis Comprehensive Care Center. Neurol Neuroimmunol Neuroinflamm. 2020; 7(5), doi: 10.1212/NXI.0000000000000835, indexed in Pubmed: 32646885.

24. Capasso N, Palladino R, Montella E, et al. Prevalence of SARS-CoV-2 Antibodies in Multiple Sclerosis: The Hidden Part of the Iceberg. J Clin Med. 2020; 9(12), doi: 10.3390/jcm9124066, indexed in Pubmed: 33339402. 
25. Sepúlveda M, Llufriu S, Martínez-Hernández E, et al. Incidence and Impact of COVID-19 in MS: A Survey From a Barcelona MS Unit. Neurol Neuroimmunol Neuroinflamm. 2021; 8(2), doi: 10.1212/ NXI.00000000000000954, indexed in Pubmed: 33504634.

26. Sahraian MA, Azimi A, Navardi S, et al. Evaluation of the rate of COVID-19 infection, hospitalization and death among Iranian patients with multiple sclerosis. Mult Scler Relat Disord. 2020; 46: 102472, doi: 10.1016/j.msard.2020.102472, indexed in Pubmed: 32890817.

27. REDONE.br - Neuroimmunology Brazilian Study Group Focused on COVID-19 and MS. Incidence and clinical outcome of Coronavirus disease 2019 in a cohort of 11,560 Brazilian patients with multiple sclerosis. Mult Scler. 2021 [Epub ahead of print]: 1352458520978354, doi: 10.1177/1352458520978354, indexed in Pubmed: 33528295.

28. Ciampi E, Uribe-San-Martin R, Cárcamo C. COVID-19 pandemic: The experience of a multiple sclerosis centre in Chile. Mult Scler Relat Disord. 2020; 42: 102204, doi: 10.1016/j.msard.2020.102204, indexed in Pubmed: 32570203.

29. Fan M, Qiu W, Bu B, et al. Risk of COVID-19 infection in MS and neuromyelitis optica spectrum disorders. Neurol Neuroimmunol Neuroinflamm. 2020; 7(5), doi: 10.1212/NXI.0000000000000787, indexed in Pubmed: 32503092.

30. Liu Y, Mao B, Liang S, et al. Shanghai Clinical Treatment Experts Group for COVID-19. Association between age and clinical characteristics and outcomes of COVID-19. Eur Respir J. 2020; 55(5), doi: 10.1183/13993003.01112-2020, indexed in Pubmed: 32312864.

31. Wolff D, Nee S, Hickey NS, et al. Risk factors for Covid-19 severity and fatality: a structured literature review. Infection. 2021; 49(1): 15-28, doi: 10.1007/s15010-020-01509-1, indexed in Pubmed: 32860214.

32. Olloquequi J. COVID-19 Susceptibility in chronic obstructive pulmonary disease. Eur J Clin Invest. 2020; 50(10): e13382, doi: 10.1111/ eci.13382, indexed in Pubmed: 32780415.

33. Zhou Y, Yang Q, Chi J, et al. Obesity and diabetes as high-risk factors for severe coronavirus disease 2019 (Covid-19). Diabetes Metab Res Rev. 2021; 37(2): e3377-56, doi: 10.1002/dmrr.3377, indexed in Pubmed: 32588943.

34. Kronbichler A, Kresse D, Yoon S, et al. Asymptomatic patients as a source of COVID-19 infections: A systematic review and meta-analysis. Int J Infect Dis. 2020; 98: 180-186, doi: 10.1016/j.jijd.2020.06.052, indexed in Pubmed: 32562846.

35. Grant M, Geoghegan L, Arbyn M, et al. The Prevalence of Symptoms in 24,410 Adults Infected by the Novel Coronavirus (SARS-CoV-2; COVID-19): A Systematic Review and Meta-Analysis of 148 Studies from 9 Countries. SSRN Electronic Journal. , doi: 10.2139/ssrn.3582819.
36. Mycko M. B cell targeting therapies in MS patients during the SARS-CoV-2 pandemic - when immunosuppression meets infection? Neurologia i Neurochirurgia Polska. 2020; 54(6): 490-501, doi: 10.5603/ pjnns.a2020.0083.

37. Hughes R, Pedotti R, Koendgen H. COVID-19 in persons with multiple sclerosis treated with ocrelizumab - A pharmacovigilance case series. Mult Scler Relat Disord. 2020; 42: 102192, doi: 10.1016/j. msard.2020.102192, indexed in Pubmed: 32570202.

38. Sormani MP, De Rossi N, Schiavetti I, et al. Musc-19 Study Group. Disease-Modifying Therapies and Coronavirus Disease 2019 Severity in Multiple Sclerosis. Ann Neurol. 2021; 89(4): 780-789, doi: 10.1002/ana.26028, indexed in Pubmed: 33480077.

39. Sharifian-Dorche M, Sahraian MA, Fadda G, et al. COVID-19 and disease-modifying therapies in patients with demyelinating diseases of the central nervous system: A systematic review. Mult Scler Relat Disord. 2021 [Epub ahead of print]; 50: 102800, doi: 10.1016/j.msard.2021.102800, indexed in Pubmed: 33578206.

40. Adamczyk-Sowa M, Adamczyk B, Kułakowska A, et al. Secondary progressive multiple sclerosis - from neuropathology to definition and effective treatment. Neurologia i Neurochirurgia Polska. 2020; 54(5): 384-398, doi: 10.5603/pjnns.a2020.0082.

41. Loonstra FC, Hoitsma E, van Kempen ZLE, et al. COVID-19 in multiple sclerosis: The Dutch experience. Mult Scler. 2020; 26(10): 12561260, doi: $10.1177 / 1352458520942198$, indexed in Pubmed: 32662742.

42. Dalla Costa G, Leocani L, Montalban X, et al. RADAR-CNS consortium. Real-time assessment of COVID-19 prevalence among multiple sclerosis patients: a multicenter European study. Neurol Sci. 2020 41(7): 1647-1650, doi: 10.1007/s10072-020-04519-x, indexed in Pubmed: 32617741.

43. Haji Abdolvahab M, Moradi-Kalbolandi S, Zarei M, et al. Potential role of interferons in treating COVID-19 patients. Int Immunopharmacol. 2021; 90: 107171, doi: 10.1016/j.intimp.2020.107171, indexed in Pubmed: 33221168.

44. Lopinavir/ Ritonavir, Ribavirin and IFN-beta Combination for nCoV Treatment. Case Medical Research. 2020, doi: 10.31525/ct1-nct04276688.

45. Ciardi MR, Zingaropoli MA, Pasculli P, et al. The peripheral blood immune cell profile in a teriflunomide-treated multiple sclerosis patient with COVID-19 pneumonia. J Neuroimmunol. 2020 [Epub ahead of print]; 346: 577323, doi: 10.1016/j.jneuroim.2020.577323, indexed in Pubmed: 32688146. 